\title{
MULTISYSTEM INFLAMMATORY SYNDROME IN CHILDREN: DIAGNOSTIC MARKERS AND FEATURES OF PHARMACOTHERAPY
}

\author{
${ }^{1}$ Khaitovych M.V. https://orcid.org/0000-0001-6412-3243 \\ ${ }^{2}$ Voroniuk L.M. https://orcid.org/0000-0002-6687-0573 \\ ${ }^{2}$ Borisova G. Yu. https://orcid.org/0000-0002-9041-5669 \\ ${ }^{2}$ Diudenko NV. https://orcid.org/0000-0002-7851-1017 \\ ${ }^{2}$ Miagka N.M. https://orcid.org/0000-0001-5904-0603 \\ ${ }^{1}$ Bogomolets National Medical University, Kyiv, Ukraine \\ ${ }^{2} \mathrm{KNP}$ «Kyiv City Children's Clinical Hospital No. 2», Kyiv, Ukraine \\ nik3061@gmail.com
}

\begin{abstract}
Relevance. In 2020, children were hospitalized with fever and multisystem inflammation throughout the world during the COVID-19 pandemic. In the United States, this condition is called MIS-C (Multisystem Inflammatory Syndrome in Children). This syndrome is thought to be similar to the severe course of COVID-19 in adults (cytokine storm).

The objective of the work is to evaluate the features of the course and pharmacotherapy of multisystem inflammatory syndrome in children.

Materials and methods. The study included 17 children (10 boys and 7 girls) aged 3-16 years (on average $-9.5 \pm 3.4$ years). Diagnosis of coronavirus infection was performed by polymerase chain reaction with real-time detection, determined the level of immunoglobulins $\mathrm{M}$ and $\mathrm{G}$ before coronavirus infection.

Results. The duration of fever in patients was 5-21 days (average 8.1 \pm 4.0 days), the duration of inpatient treatment - 7-35 days (average $15.7 \pm 7.0$ days). Blood albumin levels were reduced in $53.8 \%$ of children; the level of fibrinogen was increased in $88.2 \%$ of children, the level of C-reactive protein, ferritin and D-dimer - in all patients. 15 (88.2\%) children had pathology of the digestive system, $13(76.5 \%)$ - cardiovascular system (7 children were diagnosed with carditis, 2 - dilation of coronary arteries, 7 - cardiac arrhythmia). Acute respiratory distress -syndrome was found in a 13-year-old girl, shock - in an 11-year-old boy, 11 children (64.7\%) were diagnosed with pathology of the respiratory system (pleurisy, pneumonia), skin and mucous membranes, and 4 children (23.5\%) there were manifestations of central nervous system disorders (meningism, decreased reflexes, ataxia), in 2 (11.8\%) - renal failure. On average, each patient had lesions of $3.9 \pm 1.2$ systems.

Conclusions. MIS-C was manifested by prolonged fever, high levels of laboratory markers of inflammation, hypoalbuminemia, hypercoagulation, often - pathological manifestations of the cardiovascular, digestive, respiratory systems, skin and mucous membranes. The treatment included intravenous immunoglobulin, steroids, anticoagulant and antibacterial therapy and was effective.

Key words: multisystem inflammatory syndrome, children.
\end{abstract}

Relevance. In 2020, hospitalization of children who developed fever and multisystem inflammation during the COVID-19 pandemic was registered in different countries of the world. Although most children had a mild course of COVID-19 infection [9], some were hospitalized in critical condition with shock and multiorgan failure and required intensive care, some had symptoms similar to those seen in Kawasaki syndrome (but the disease was accompanied by shock). In the United States, this condition is called MIS-C (multisystem inflammatory syndrome in children), in the UK - PIMS-TS (pediatric inflammatory multisystem syndrome temporally associated with COVID-19) [1] The definition states that this syndrome is associated with coronavirus infection over time. Thus, many children who developed this syndrome were found to have antibodies to SARS-CoV-2 [12], although virus tests did not always show a positive result. Some doubts about the association of this syndrome with COVID-19 infection were due to the fact that only in a small number of patients the manifestations of MIS-C were detected against the background of acute SARS-CoV-2 - this led to think that these are manifestations of various diseases [3].

Over the past six months, both individual cases of the disease $[10,11]$ and literature reviews have been published and the results of many individual studies have been summarized. It has been proven that multisystem inflammatory syndrome often occurs in the late stages of the disease, can be observed in children who were previously completely healthy, 3-4 weeks after asymptomatic infection.

It is believed that this syndrome is analogous to the severe course of COVID-19 in adults (cytokine storm). It is caused by the body's excessive immune response to COVID-19. This develops a variant of virus-induced secondary hemophagocytic syndrome [7], which is massive, uncontrolled, often fatal activation of the immune system, the release of pro-inflammatory cytokines (TNF-alpha, IL-1, IL-2 IL-6, IL-8, IL -10, 
granulocyte colony-stimulating factor, monocyte chemoattractive protein-1 and markers of inflammation (C-reactive protein, serum ferritin), which can lead to the development of acute respiratory distress syndrome, multiple organ failure and can cause patient death.

Mostly MIS-C was found in children aged 3-12 years, less common in adolescents and young children, but also described in newborns [2]. There were mainly lesions of the heart, blood vessels, kidneys, digestive system, brain, skin and eyes. The following symptoms are typical: fever that lasts more than a day; feeling tired; intense abdominal pain, which may be accompanied by vomiting and/or diarrhea; chest pain; rapid heartbeat; dyspnea; lowering blood pressure; cyanosis of the lips or face; «Brain fog», skin rash; redness of the eyes; swelling of the extremities, lips and tongue. Thus, according to Indian researchers, $100 \%$ of children with this syndrome have a fever, $80 \%$ - neurological disorders, half pathology of the lower respiratory tract, $35 \%$ - rash, $25 \%$ - acute gastroenteritis [5].

The objective of the work is to evaluate the features of the course and pharmacotherapy of multisystem inflammatory syndrome in children.

\section{MATERIALS AND METHODS}

The study included 17 children (10 boys and 7 girls) aged 3-16 years (on average $-9.5 \pm 3.4$ years). All patients were of normal physical development (average body mass index was $18.8 \pm 2.8 \mathrm{~kg} / \mathrm{m} 2$ ).

The examination plan included a general clinical study, also determined the levels of albumin, C-reactive protein, ferritin, fibrinogen, procalcitonin, D-dimer in plasma. Ultrasound examination was performed using a MyLab X6 device according to a standard protocol. General clinical blood test was performed on an automatic hematological analyzer SwelabAlfa, MedonicM-series, Quintus.

Diagnosis of coronavirus infection was performed by polymerase chain reaction with real-time detection using a test system for COVID-19 (Korea). Determination of immunoglobulins $\mathrm{M}$ and $\mathrm{G}$ for coronavirus infection, D-dimer was performed on an automatic immunofluorescence analyzer miniVIDAS, coagulogram - on a semi-automatic hematological coagulometer CA52.

Statistical processing of the research results was carried out using a licensed office suite Microsoft Excel 2010 and SPSS 22.3. The results were considered reliable at $\mathrm{p}<0.05$.

\section{RESULTS AND DISCUSSION}

In most cases, children complained (Table 1) of a prolonged increase in body temperature (body temperature above $38.0^{\circ} \mathrm{C}$ was registered in 1 child, above $38.5^{\circ} \mathrm{C}-$ in the remaining 16 children); abdominal pain (88.2\%); nausea (82.3\%); vomiting (70.6\%); diarrhea (29.4\%); muscle weakness $(82.3 \%)$; skin rash $(64.7 \%)$; redness of the eyes $(70.6 \%)$, difficulty breathing $(17.6 \%)$.

Table 1

Frequency (\%) of complaints in children with MIS-C

\begin{tabular}{|c|c|c|}
\hline \multirow{2}{*}{ Complaint } & \multicolumn{2}{|c|}{ The number of children } \\
\cline { 2 - 3 } & absolute & \% \\
\hline Headache & 9 & 52,9 \\
\hline Muscle weakness & 14 & 82,3 \\
\hline Abdominal pain & 15 & 88,2 \\
\hline Nausea & 14 & 82,3 \\
\hline Vomiting & 12 & 70,6 \\
\hline Diarrhea & 5 & 29,4 \\
\hline Conjunctivitis & 12 & 70,6 \\
\hline Skin rash & 11 & 64,7 \\
\hline Difficulty breathing & 3 & 17,6 \\
\hline
\end{tabular}

According to the results of objective and instrumental research (Table 2), in 15 (88.2\%) children revealed pathology of the digestive system. 13 children (76.5\%) were diagnosed with cardiovascular lesions, including 7 children with carditis, 2 children with dilation of coronary arteries, and 7 with cardiac arrhythmia. Acute respiratory distress syndrome was detected in 1 child (13-year-old girl), shock - in an 11-year-old boy. Eleven children (64.7\%) were diagnosed with pathology of the respiratory system (pleurisy, pneumonia), skin and mucous membranes. In 4 children $(23.5 \%)$ there were manifestations of central nervous system disorders (meningism, decreased reflexes, ataxia), in 2 children (11.8\%) - renal failure.

On average, each patient had lesions of $3.9 \pm 1.2$ systems, often with a combination of lesions of the digestive tract, skin and mucous membranes, cardiovascular and respiratory disorders.

Generally, the level of fibrinogen was increased in $88.2 \%$ of children, the level of C-reactive protein, ferritin and D-dimer was increased in all patients. The average values of laboratory parameters are shown in table 3 . The average level of ferritin was $403,0 \pm 314,3$ $\mathrm{ng} / \mathrm{ml}$, C-reactive protein $-75,4 \pm 56,7 \mathrm{mg} / \mathrm{l}$, D-dimer

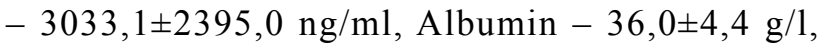
Fibrinogen $-4,9 \pm 1,7 \mathrm{~g} / \mathrm{l}$, Procalcitonin $-2,6 \pm 4,5 \mathrm{ng} / \mathrm{ml}$.

The level of blood albumin was reduced in $53.8 \%$ of children and was at the level of 27-37 g/l, while in other patients the level of albumin, although within the age limit, but close to the lower limit (38-43 g/l), a significant $(p<0.05)$ negative correlation was found between the level of albumin in the blood and the duration of the fever period $(\mathrm{r}=-0.71)$.

The treatment of 11 children included intravenous immunoglobulin, all children - dexamethasone, 10 children - methylprednisolone, 14 children acetylsalicylic acid, 1 child - epinephrine, 1 child heparin, 5 children - enoxyparin. 
Table 2

Frequency $(\%)$ of lesions of different systems in children with MIS-C

\begin{tabular}{|l|c|c|}
\hline \multirow{2}{*}{ Affected system } & \multicolumn{2}{c|}{ Number of children } \\
\cline { 2 - 3 } & absolute & $\%$ \\
\hline $\begin{array}{l}\text { Cardiovascular (carditis, pericarditis, } \\
\text { cardiac arrhythmias) }\end{array}$ & 13 & 76,5 \\
\hline $\begin{array}{l}\text { Respiratory (shortness of breath, } \\
\text { pneumonia, pleurisy) }\end{array}$ & 11 & 64,7 \\
\hline Shock & 1 & 5,9 \\
\hline Acute respiratory distress syndrome & 1 & 5,9 \\
\hline $\begin{array}{l}\text { Digestive (abdominal pain, loss of } \\
\text { appetite, nausea, vomiting) }\end{array}$ & 15 & 88,2 \\
\hline Renal failure & 2 & 11,8 \\
\hline Central nervous (ataxia, meningism) & 4 & 23,5 \\
\hline Skin and mucous membranes & 11 & 64,7 \\
\hline
\end{tabular}

Table 3

Average values $(\mathrm{X} \pm \mathrm{SD})$ of laboratory parameters of children with MIS-C

\begin{tabular}{|l|c|}
\hline \multicolumn{1}{|c|}{$\begin{array}{c}\text { Characteristics of the examined } \\
\text { children }\end{array}$} & Average value $(\mathbf{X} \pm \mathbf{S D})$ \\
\hline Ferritin, $\mathrm{ng} / \mathrm{ml}$ & $403,0 \pm 314,3$ \\
\hline C-reactive protein, $\mathrm{mg} / \mathrm{l}$ & $75,4 \pm 56,7$ \\
\hline D-dimer, $\mathrm{ng} / \mathrm{ml}$ & $3033,1 \pm 2395,0$ \\
\hline Albumin, g/1 & $36,0 \pm 4,4$ \\
\hline Procalcitonin, $\mathrm{ng} / \mathrm{ml}$ & $2,6 \pm 4,5$ \\
\hline Fibrinogen, $\mathrm{g} / \mathrm{l}$ & $4,9 \pm 1,7$ \\
\hline Neutrophils $\times 10^{9} / 1$ & $8,3 \pm 4,6$ \\
\hline Lymphocytes $\times 10^{9} / 1$ & $1,6 \pm 1,0$ \\
\hline
\end{tabular}

Considering the risk of bacterial infection, all patients received antibiotic therapy, mainly 3rd generation cephalosporins: 13 patients received ceftriaxone, including 9 as monotherapy, 2 children - ceftazidime and cefixime, 1 child was included in the treatment regimen of cefipime, 3 children received amikacin, 3 children - vancomycin, 2 children - levofloxacin.

The duration of fever in the examined patients was 5-21 days (average 8.1 \pm 4.0 days the length of stay in hospital was 7-35 days (on average 15.7 \pm 7.0 days).

Thus, we considered the course of a new disease in children associated with the SARS-CoV-2 virus. The syndrome involves many organs: the heart, lungs, gastrointestinal tract, nervous system, skin and mucous membranes. The results of our study coincide with those previously described in the literature [8]. We confirmed that this condition is mainly observed in children aged $3-12$ years $(76.5 \%)$ and in all patients laboratory manifestations of inflammation and hypercoagulation (sharply elevated levels of C-reactive protein, ferritin, fibrinogen and $\mathrm{D}$-dimer). We found a close inverse correlation between the length of the fever and the blood albumin levels of children with
MIS-C. Other researchers have also found low albumin levels in MIS-C [6].

It should be noted that in clinics abroad, the complex treatment of MIS-C patients included an interleukin-1 receptor antagonist anakinra [4], interleukin-6 receptor blockers (example tocilizumab) and a tumor necrotic factor antagonist (example infliximab). Our experience has confirmed the effectiveness of intravenous immunoglobulin, dexamethasone, anticoagulant therapy in the treatment of children with multisystem inflammatory syndrome.

\section{CONCLUSIONS}

Multisystem inflammatory syndrome in children is an analogue of the cytokine storm in COVID-19 infection in adults. The disease is manifested by prolonged fever, high levels of laboratory markers of inflammation, hypoalbuminemia, hypercoagulation, often pathological manifestations of the cardiovascular, digestive and respiratory systems, skin and mucous membranes. The complex of treatment, which included intravenous immunoglobulin, steroids, anticoagulant and antibacterial therapy, proved to be effective.

\section{REFERENCES}

1. Ahmed M., Advani S., Moreira A., Zoreti S., Martinez J. et al. Multisystem inflammatory syndrome in children: A systematic review. EClinicalMedicine. 2020 Sep 01; 26: 100527. DOI: 10.1016/j.eclinm.2020.100527.

View at: Publisher Site: https://www.thelancet.com/ journals/eclinm/article/PIIS2589-5370(20)30271-6/ fulltext

Scholar: https://scholars.uthscsa.edu/en/publications/ multisystem-inflammatory-syndrome-in-children-asystematic-review

Europe PMC: https://europepmc.org/article/ $\mathrm{med} / 32923992$

2. Borkotoky R.K., Barua P.B., Paul S.P., Heaton P.A. COVID-19-related Potential Multisystem Inflammatory Syndrome in Childhood in a Neonate Presenting as Persistent Pulmonary Hypertension of the Newborn. Pediatr Infect Dis J. 2021 Jan 12. Online ahead of print. DOI: 10.1097/inf.0000000000003054

View at: Publisher Site: https://journals.lww.com/ pidj/Abstract/9000/COVID_19_related_Potential_ Multisystem.95900.aspx

PubMed: https://pubmed.ncbi.nlm.nih.gov/33464010/ Europe PMC: https://europepmc.org/article/ $\operatorname{med} / 33464010$

3. Esposito S., Principi N. Multisystem Inflammatory Syndrome in Children Related to SARS-CoV-2. Paediatr Drugs. 2021 Jan 22; 1-11. Online ahead of print. DOI: 10.1007/s40272-020-00435-x.

View at: Publisher Site: https://link.springer.com/ article/10.1007\%2Fs40272-020-00435-X

4. Fouriki A., FougèreY., De Camaret C., Rohner 
G.B., Grazioli S., Wagner N. et al. Case Report: Case Series of Children With Multisystem Inflammatory Syndrome Following SARS-CoV-2 Infection in Switzerland. Front Pediatr. 2021 Jan 5; 8: 594127. DOI: 10.3389/fped.2020.594127.

View at: Publisher Site: https://www.frontiersin.org/ articles/10.3389/fped.2020.594127/full

PubMed: https://pubmed.ncbi.nlm.nih.gov/33469522/ PubMed Central: https://www.ncbi.nlm.nih.gov/pmc/ articles/PMC7813982/

5. Gupta S., Chopra N., Singh A., Gera R., Chellani H. Pandey R., Arora B.S. Unusual Clinical Manifestations and Outcome of Multisystem Inflammatory Syndrome in Children (MIS-C) in a Tertiary Care Hospital of North India. J Trop Pediatr. 2021 Jan 29; 67(1): fmaa127. DOI: 10.1093/tropej/fmaa127

View at: Publisher Site: https://academic.oup.com/ tropej/article-abstract/67/1/fmaa127/6123399?redirec tedFrom=fulltext

PubMed: https://pubmed.ncbi.nlm.nih.gov/33513240/ Europe PMC: https://europepmc.org/article/ $\operatorname{med} / 33513240$

6. Kest H., Kaushik A., DeBruin W., Colletti M., Goldberg D. Multisystem Inflammatory Syndrome in Children (MIS-C) Associated with 2019 Novel Coronavirus (SARS-CoV-2) Infection. Case Reports in Pediatrics. 2020. 2020: Article ID 8875987, 4 pages. DOI: $10.1155 / 2020 / 8875987$

View at: Publisher Site: https://www.hindawi.com/ journals/cripe/2020/8875987/

PubMed: https://pubmed.ncbi.nlm.nih.gov/32733733/

PubMed Central: https://www.ncbi.nlm.nih.gov/pmc/ articles/PMC7383305/

7. Kwak J.H., Lee S.-Y., Choi J.-W. Korean Society of Kawasaki Disease. 2020. Clinical features, diagnosis, and outcomes of multisystem inflammatory syndrome in children associated with coronavirus disease. 2019. Clinical and Experimental Pediatrics 2021; 64(2): 6875. DOI: $10.3345 /$ cep. 2020.01900

View at: Publisher Site: https://www.e-cep.org/journal/ view.php?doi=10.3345/cep.2020.01900

PubMed: https://pubmed.ncbi.nlm.nih.gov/33445833/

PubMed Central: http://www.ncbi.nlm.nih.gov/pmc/ articles/pmc7873390/

Europe PMC: https://europepmc.org/article/ $\mathrm{med} / 33445833$

8. Mehra B., Aggarwal V., Kumar P., Kundal M., Gupta D., Kumar A. et al. COVID-19-associated Severe Multisystem Inflammatory Syndrome in Children with Encephalopathy and Neuropathy in an Adolescent Girl with the Successful Outcome: An Unusual Presentation. Indian J Crit Care Med. 2020; 24(12): 1276-8. DOI: 10.5005/jp-journals-10071-23685
View at: Publisher Site: https:/www.ijccm.org/doi/ pdf/10.5005/jp-journals-10071-23685

PubMed: https://pubmed.ncbi.nlm.nih.gov/33446986/ PubMed Central: https://www.ncbi.nlm.nih.gov/ research/coronavirus/publication/33446986

9. Müller J., Oberhoffer R., Brudy L., Ewert P. COVID-19 and paediatric patient involvement (cardiovascular aspects). Eur Heart J Suppl. 2020 Dec 23; 22(Suppl Pt t): P19-P24. DOI: 10.1093/eurheartj/suaa168

View at: Publisher Site: https://academic.oup.com/ eurheartjsupp/article/22/Supplement_P/P19/6046108 PubMed: https://pubmed.ncbi.nlm.nih.gov/33390865/ PubMed Central: http://www.ncbi.nlm.nih.gov/pmc/ articles/pmc7757714/

Europe PMC: https://europepmc.org/article/ $\mathrm{med} / 33390865$

10. Mustafa M.R., Carter M.J., Wong J., Bell A., Salih C. Coronary aneurysms, myocardial dysfunction, and shock in a COVID-19 child, role of ECMO, immunomodulation, and cardiac CT. Cardiol Young. 2021 Jan 28; 1-5. DOI: 10.1017/ s1047951121000147

View at: Publisher Site: https://www.cambridge. org/core/journals/cardiology-in-the-young/ article/abs/coronary-aneurysms-myocardialdysfunction-and-shock-in-a-covid19-childrole-of-ecmo-immunomodulation-and-cardiacct/49B5F4C159FB2FDE1D63B9C30C1C085D

PubMed: https://pubmed.ncbi.nlm.nih.gov/33504377/ Europe PMC: https://europepmc.org/article/ $\mathrm{med} / 33504377$

11. Navaeifar M. R., Shahbaznejad L., Lotfabadi A.S., Rezai M.S. COVID-19-Associated Multisystem Inflammatory Syndrome Complicated with Giant Coronary Artery Aneurysm. Case Rep Pediatr. 2021; 2021: Article ID 8836403. DOI: $10.1155 / 2021 / 8836403$ View at: Publisher Site: https://www.hindawi.com/ journals/cripe/2021/8836403/

12. Schvartz A., Belot A., Kone-Paut I. Pediatric Inflammatory Multisystem Syndrome and Rheumatic Diseases During SARS-CoV-2 Pandemic. Front Pediatr. 2020; 8: 605807. DOI: 10.3389/ fped.2020.605807.

View at: Publisher Site: https://www.frontiersin.org/ articles/10.3389/fped.2020.605807/full

PubMed: https://pubmed.ncbi.nlm.nih.gov/33344389/ PubMed Central: http://www.ncbi.nlm.nih.gov/pmc/ articles/pmc7746854/

Europe PMC: https://europepmc.org/article/ $\mathrm{med} / 33344389$

Article history Received: 13.02 .2021 Revision requested: 21.02 .2021 Revision received: 28.02 .2021 Accepted: 29.03 .2021 Accepted: 29.03 .2021 


\title{
МУЛЬТИСИСТЕМНИЙ ЗАПАЛЬНИЙ СИНДРОМ У ДІТЕЙ: МАРКЕРИ ДІАГНОСТИКИ ТА ОСОБЛИВОСТІ ФАРМАКОТЕРАПІЇ
}

\author{
${ }^{1}$ Хайтович М.В., ${ }^{2}$ Воронюк Л.М., ${ }^{2}$ Борисова Г.Ю., ${ }^{2}$ Дюденко Н.В., ${ }^{2}$ Мягка Н.М. \\ ${ }^{1}$ Національний медичний університет імені О.О. Богомольия, Киї, Україна \\ ${ }^{2}$ КНП «Київська міська дитяча клінічна лікарня № 2», Київ, Украӥна \\ nik3061@gmail.com
}

Актуальність. У 2020 році у різних країнах світу зареєстровано госпіталізацію дітей, у яких в період пандемії COVID-19 виникла лихоманка і мультисистемне запалення. У США даний стан отримав назву MIS-C (multisystem inflammatory syndrome in children). Вважається, що даний синдром є аналогом тяжкого перебігу COVID-19 у дорослих (цитокіновий шторм).

Мета - оцінити особливості перебігу та фармакотерапії мультисистемного запального синдрому у дітей.

Матеріали та методи. Під наглядом знаходилось 17 дітей (10 хлопчиків та 7 дівчаток) віком 3-16 років (в середньому -

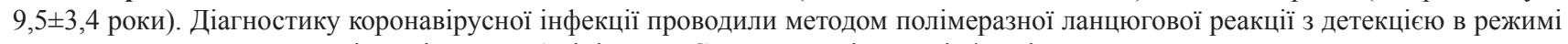
реального часу, визначали рівень імуноглобулінів М та G до коронавірусної інфекції.

Результати. Тривалість лихоманки у пацієнтів становила 5-21 добу (в середньому 8,1 4,0 діб), тривалість перебування на стаціонарному лікуванні - 7-35 діб (в середньому 15,7 7,0 діб). Рівень альбуміну крові був знижений у 53,8\% дітей; рівень фібриногену був підвищений у 88,2\% дітей, рівень С-реактивного білка, феритину та D-димеру - у всіх пацієнтів. У 15 (88,2\%) дітей виявлено патологію травної системи, у 13 (76,5\%) - серцево-судинної системи (у 7 дітей діагностовано кардит, у 2 - розширення коронарних артерій, у 7 - аритмію серця. Гострий респіраторний дистрес-синдром виявлено у 13-річної дівчинки, шок - у 11-річного хлопчика. У 11 дітей (64,7\%) діагностовано патологію дихальної системи (плеврит, пневмонію тощо), шкіри та слизових оболонок. У 4 дітей $(23,5 \%)$ відмічено прояви порушення центральної нервової системи (менінгізм, зниження рефлексів, атаксію), у 2 (11,8\%) - ниркової недостатності. В середньому у кожного пацієнта було відмічено ураження $3,9 \pm 1,2$ систем.

Висновки. MIS-C виявлявся тривалою лихоманкою, високими рівнями лабораторних маркерів запалення, гіпоальбумінемією, гіперкоагуляцією, часто - патологічними проявами з боку серцево-судинної, травної, дихальної систем, шкіри та слизових оболонок. Комплекс лікування влючав імуноглобулін внутрішньовенно, стероїди, антикоагулянтну та антибактеріальну терапію і був ефективним.

Ключові слова: мультисистемний запальний синдром, діти.

\section{МУЛЬТИСИСТЕМНЫЙ ВОСПАЛИТЕЛЬНЫЙ СИНДРОМ У ДЕТЕЙ: МАРКЕРЫ ДИАГНОСТИКИ И ОСОБЕННОСТИ ФАРМАКОТЕРАПИИ}

\author{
${ }^{1}$ Хайтович Н.В., ${ }^{2}$ Воронюк Л.М., ${ }^{2}$ Борисова Г.Ю., ${ }^{2}$ Дюденко Н.В., ${ }^{2}$ Мягкая Н.Н. \\ 1 Национальный медицинский университет имени А.А. Богомольца, Киев, Украина \\ 2КНП «Киевская городская детская клиническая больница № 2», Киев, Украина \\ nik3061@gmail.com
}

\begin{abstract}
Актуальность. В 2020 году в разных странах мира зарегистрирована госпитализация детей, у которых в период пандемии COVID-19 возникла лихорадка и мультисистемное воспаление. В США данное состояние получило название MIS-C (multisystem inflammatory syndrome in children). Считается, что данный синдром является аналогом тяжелого течения COVID-19 у взрослых (цитокиновый шторм).

Цель - оценить особенности течения и фармакотерапии мультисистемного воспалительного синдрома у детей.

Материалы и методы. Под наблюдением находилось 17 детей (10 мальчиков и 7 девочек) в возрасте 3-16 лет (в среднем - 9,5 $\pm 3,4$ года). Диагностику коронавирусной инфекции проводили методом полимеразной цепной реакции с детекцией в режиме реального времени, определяли уровень иммуноглобулинов $\mathrm{M}$ и $\mathrm{G}$ к коронавирусной инфекции.

Результаты. Продолжительность лихорадки у обследованных пациентов составила 5-21 суток (в среднем 8,1 $\pm 4,0$ суток), продолжительность пребывания на стационарном лечении - 7-35 суток (в среднем $15,7 \pm 7,0$ суток). Уровень альбумина крови был снижен у $53,8 \%$ детей; уровень фибриногена был повышен у $88,2 \%$ детей, уровень C-реактивного белка, ферритина и D-димера - у всех пациентов. У 15 (88,2\%) детей выявлена патология пищеварительной системы, у 13 (76,5\%) сердечно-сосудистой системы (у 7 детей диагностирован кардит, у 2 - расширение коронарных артерий, у 7 - аритмия сердца). Острый респираторный дистресс-синдром выявлен у 13-летней девочки, шок - у 11-летнего мальчика. У 11 детей (64,7\%) диагностирована патология дыхательной системы (плеврит, пневмония и т.д.), кожи и слизистых оболочек; у 4 детей (23,5\%) виявлены нарушения центральной нервной системы (менингизм, снижение рефлексов, атаксия), у 2 (11,8\%) - проявления почечной недостаточности. В среднем, у каждого пациента было отмечено поражение $3,9 \pm 1,2$ систем.

Выводы. MIS-C выявлялся длительной лихорадкой, высокими уровнями лабораторных маркеров воспаления, гипоальбуминемией, гиперкоагуляцией, часто - патологическими проявлениями со стороны сердечно-сосудистой, пищеварительной, дыхательной систем, кожи и слизистых оболочек. Комплекс лечения влючал иммуноглобулин внутривенно, стероиды, антикоагулянтную и антибактериальную терапию и был эффективен.

Ключевые слова: мультисистемный воспалительный синдром, дети.
\end{abstract}

